\title{
Reliable Data Transmission with Sub-Packet Generation and Fusion in Wireless Sensor Networks
}

\author{
Dr. Abul Bashar \\ Prince Mohammad Bin Fahd University, \\ Kingdom of Saudi Arabia. \\ Dr. Dinesh Kumar, \\ Computer Science and Engineering, \\ Koneru Lakshmaiah Education Foundation, \\ Andra Pradesh, India.
}

\begin{abstract}
When transmitting data in a large number, the number of conflicts that arise is high. This is especially the case when there is dense distribution of marginal wireless sensor network (WSN). Some of the major conflicts that affect the overall operation of a system include heavy transmission delay and large data loss. In this proposed work, a multipath reliable transmission is used for wireless sensor network. In order to determine the reliability of the system, the WSN implements redundancy methodology. As the first step, data is sub-divided into packets of information along with data redundancy. These packets are then transmitted via multi-paths to their corresponding destination nodes. Experimental observation shows that the proposed work indicates a significant increase in network lifetime, reduction in transmission delay and data packet loss rate.
\end{abstract}

Keywords: Data redundancy, reliable transmission; concurrent braided; WSN; multi-path

\section{Introduction}

As wireless sensor network (WSN) has become a crucial part of a number of a number of applications, there is more demand ensuring a reliable and safe means of information sharing. This is especially the case for dense distribution of WSNs [1-2]. Blind zone, attenuation and on-site environment are some conditions which need to taken into consideration that may affect the transmission of information in a WSN. This will also result in packet loss issues, errors in transmission, node failure etc while sending data. To improve data transmission, data redundancy is used as a means to ensure that data is transferred in a more reliable manner. However, there is constraint on the number of times retransmission takes place since it needs to take place from end-to-end, repeating the entire process [3]. However, using retransmission, it is possible to attain reliable and secure mass data transmission between the sensor nodes and the base station. The use of data retransmission will not only ensure reliable data transmission, it will also result in an increase in communication reliability [4].

Multi-path methodology uses multiple paths to transmit the data between the base station and the source node resulting in an improved data transmission success rate [5]. On the other hand, when a single-path is used, the possibility of losing data is very high, but there is a balance between the network load as well as its fault tolerance. The trade-off with this method is lowered performance and decreased efficiency due to increase in the number of hops [6]. There are two types of multipath methodologies used namely separated multi-path and braided multi-path. A separated multi-path is preferred over the braided because it improves data traffic, transmission delay and bandwidth significantly, compromising on overhead path construction. In the proposed work we use a redundancy strategy with which the WSN is able to transmit and receive information without data loss [7. Here we first segregate WSN into clusters based on hierarchy. The data collected by the nodes are gathered and sent to the cluster head node. This collected data is further forwarded to to sink node using braided multipath. This proposed work ensures that there is a significant reduction in in data lost rate and further any improvement in network energy consumption, reduction in transmission delay and improvement in reliability of data transmission are observed [8-9].

The organisation of the paper is as follows: Section 2 gives the literature survey of related work. Section 3 projects the proposed algorithm and in Section 4 experimental results are examined. A conclusion is drawn in Section 5.

\section{Proposed Methodology}

\subsection{Network Model}

A WSN environment that is exposed to high conflict and has a large scale of operation is taken into consideration. The WSN is made up of many sensor nodes which can be further categorized into clusters of various levels using appropriate algorithms. As part of the process, data is gathered by the nodes and sent to the 
cluster head node. From the cluster head node, the gathered data is further sent to the Sink node [10]. This transmission of data from the cluster heads to the sink nodes take place in a multi-hop path. However, there is possibility of data loss during this process of data transmission due to conflicts that are induced by the many clusters [11-12]. These conflicts will result in node failure which will also lead to data loss. Hence the following properties of a WSN network model is proposed for ease of study:

1. Energy is not limited and each base station used in unique in nature.

2. Under initial conditions, the isolated nodes will not appear, as far as high density characteristics are observed.

3. The initial energy of the sensor node is a constant.

4. Unique identifiers are used to represent the sensor nodes based on data fusion capabilities.

5. Sensor nodes also possess positioning capability.

\subsection{Structural Model}

Fig1.gives the basic structure model of two examples of WSN. Each sensor node in the WSN will gather data and send it to the cluster head nodes. At this junction, the data packets are divided into a number of sub-packets that are traced with the help of a routing table. Based on the table, the sub-packets are sent to the sink nodes. Here the data that is gathered is fused together to form the final image. Once the original data packet is retrieved from the collected sub-packets, they are discarded. When cluster head generates many sub-packets, they are known as brothers. This can be defined by the equation:

$$
\mathrm{PK}_{\mathrm{i}}=\mathrm{PK}_{\mathrm{i} 1} \cup \mathrm{PK}_{\mathrm{i} 2} \cup \ldots \cup \mathrm{PK}_{\mathrm{ij}} \cup \mathrm{PK}_{\mathrm{in}}
$$

where $\mathrm{PK}_{\mathrm{i}}$ represents the packet and the brother packets are represented as $\mathrm{PK}_{\mathrm{i} 1}, \mathrm{PK}_{\mathrm{i} 2}, \mathrm{PK}_{\mathrm{ij}}, \ldots \mathrm{PK}_{\mathrm{in}}$.

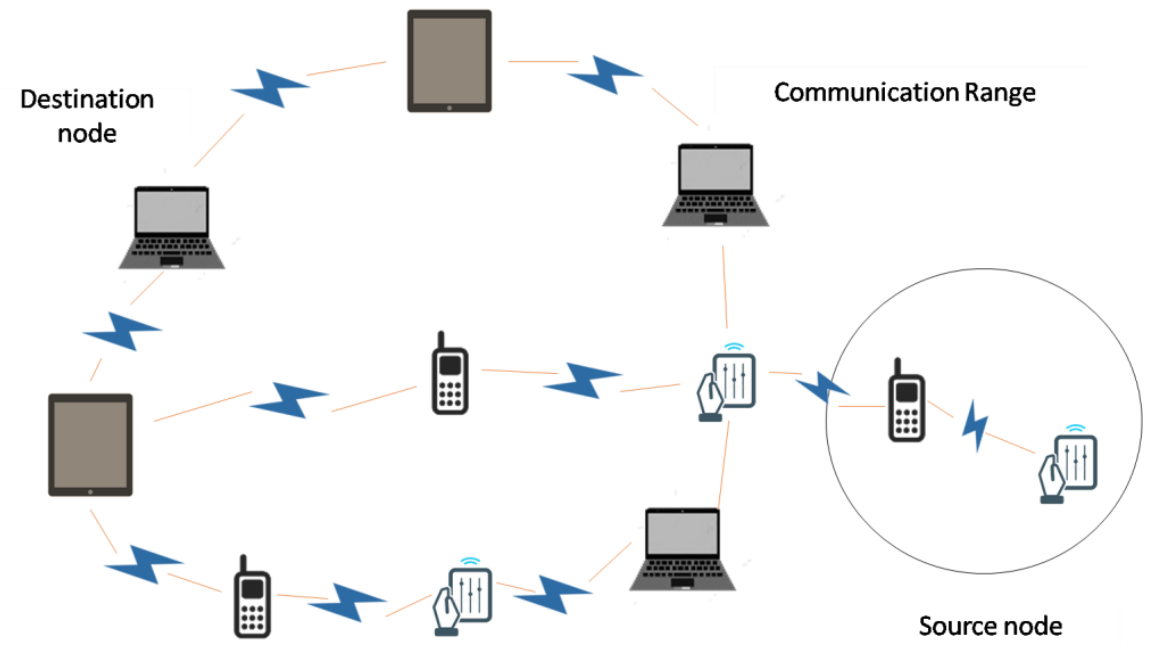

(a)

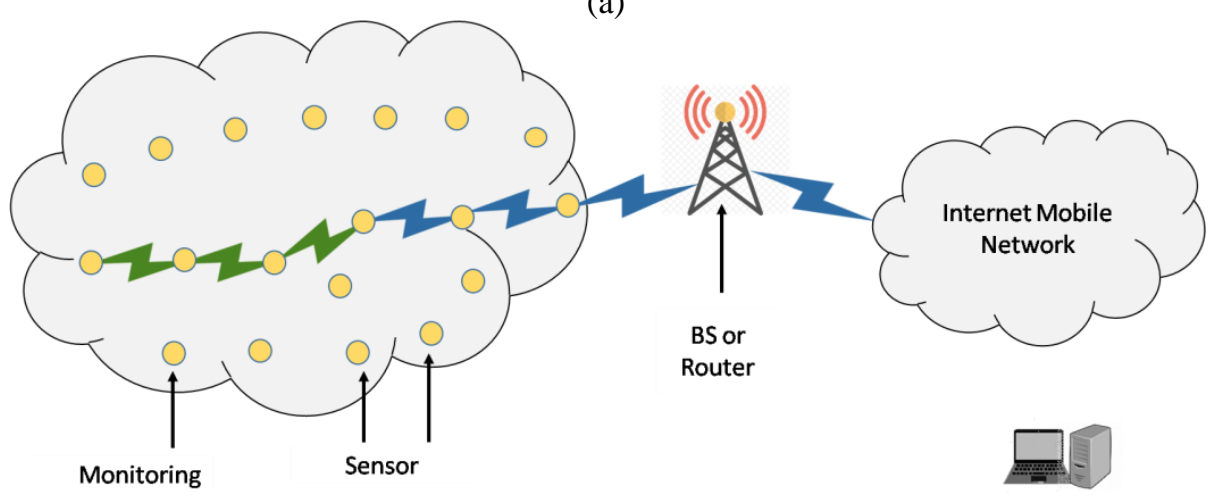

(b)

Data Processing

Fig.1. Basic structures of WSN Structural Models

ISSN: 2582-3167 


\subsection{Energy Consumption Model}

In WSN energy is consumed in three ways: when data is fused, when it is transmitted and when it is grouped. Data grouping is done by cluster head node and this will help to create many sub-packets. It has been observed that data transmission requires more energy than the other two categories. We can represent the energy required to create sub-packets from the original data using the following equation:

$$
\operatorname{Energ}_{s p}(n, x)=\frac{n(x-1)}{u} \operatorname{Energy}_{s p}
$$

where $\mathrm{n}$ is the original data, $\mathrm{x}$ represents the sub-packets and $\mathrm{u}$ is the number of bits in a data packet.

\section{Algorithm Involved}

The proposed methodology is built on network topology where the packets are sent in concurrent multi-math manner. The algorithm can be categorized into three stages:

- Data preparation: In this stage of algorithm, the original data which needs to be transmitted is first gathered and sent to the cluster head node $(\mathrm{CN})$. The data is obtained from each node and sent as brother data to the $\mathrm{CN}$.

- Data Transmission: Based on the local routing table, the brothers packets are sent into the sink in a hop-by-hop fashion. The original data is split into $\mathrm{n}$ packets, depending on the forwarding node and its size. Take into consideration two intermediate nodes which are used to transmit data to and from the cluster head nodes. We can define the relationship between digraph nodes and $\mathrm{P}$ as follows:

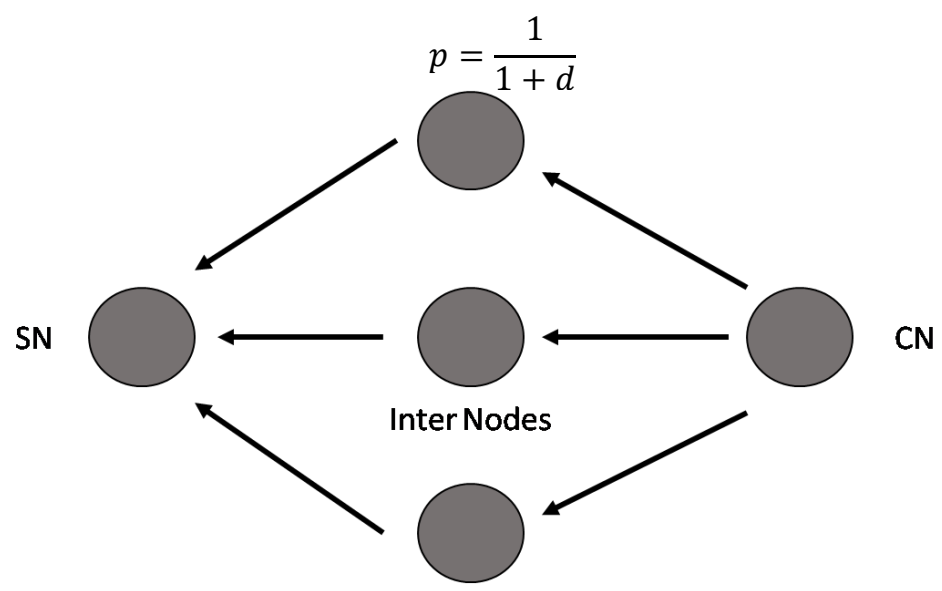

Fig.2. Data transmission Diagram

- Data fusion: At the sink node, data fusion of all the brother data packets take place. This fusion is done based on the address information that is carried by the data packets. If any redundant data is present, it will discard that packet and will carry out the rest of the process. If the sub-packets are not equal, they will be made to wait in the queue till the other packet arrive. In case any packets go amiss, a request will be sent to retransmit that particular packet.

\section{Results and Discussion}

In Fig. 3 it is observed that the proposed methodology will requires lesser amount of energy when compared to the other methodologies. Hence there is a significant reduction in cluster head node's energy cost. Moreover, single point fusion (SPF) which is considered to be a major issue in data transmission is also eliminated in this methodology. 
J. Sustain. Wireless Syst., vol. 02, no. 4, pp. 160-164

https://doi.org/10.36548/jsws.2020.4.004

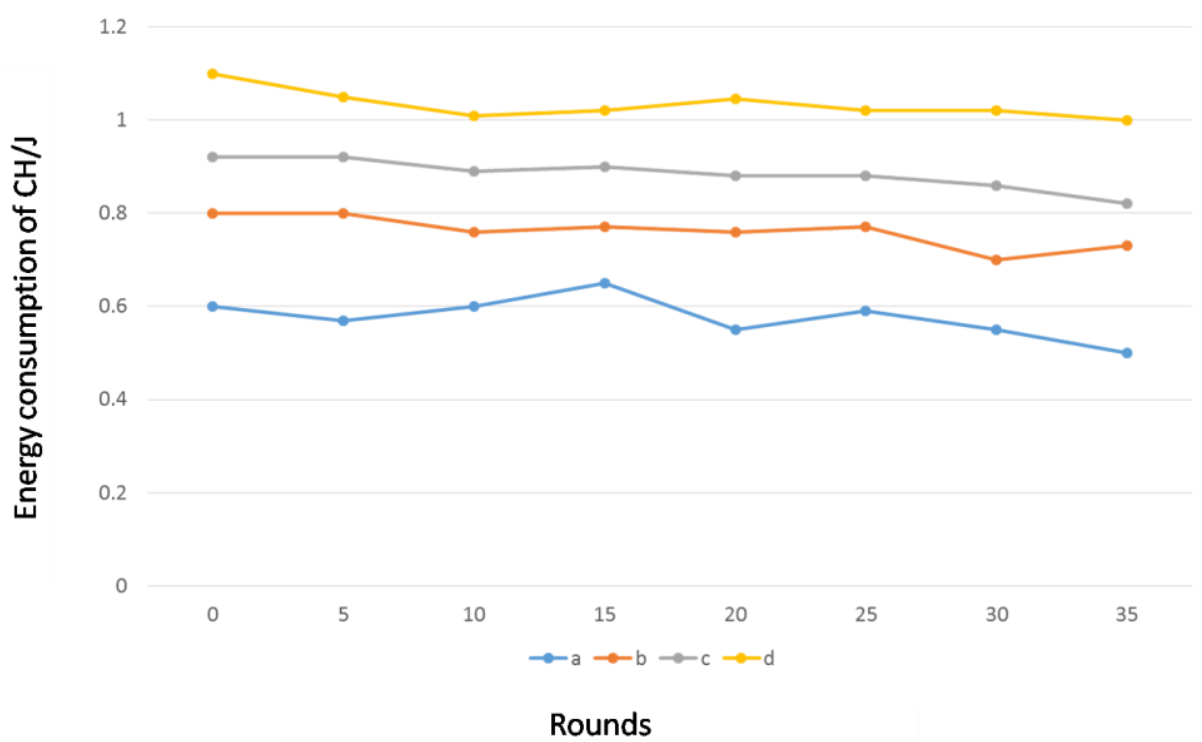

Fi.3. Energy Consumption by the Cluster Head

Similarly, in Fig.4, the number of surviving nodes that are present in the proposed system is larger than those present in other previous methodologies. During the execution process, it was observed that there is a significant increase in the clusters isolated because of the death of many cluster heads. Due to this, the network life cycle will also be reduced. In Fig.5, the data that is being transmitted from the nodes to the CNs should use either of the three protocols to reach the sink node. During this process, the shortest route is identified and used to transfer the information.

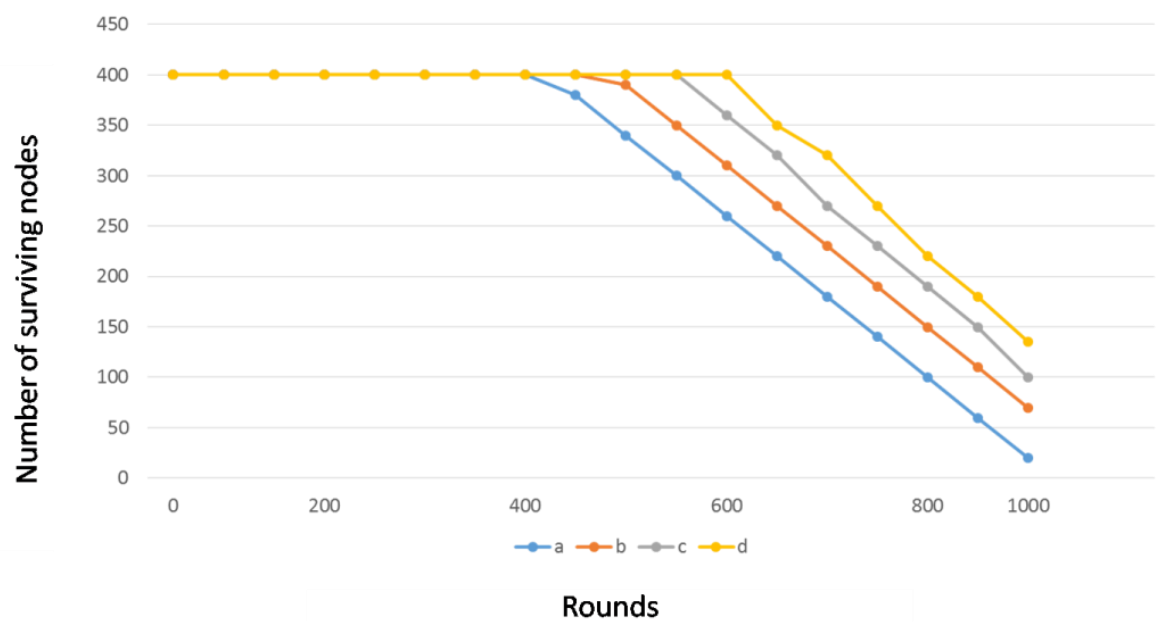

Fig.4. The total number of surviving nodes representation 
J. Sustain. Wireless Syst., vol. 02, no. 4, pp. 160-164

https://doi.org/10.36548/jsws.2020.4.004

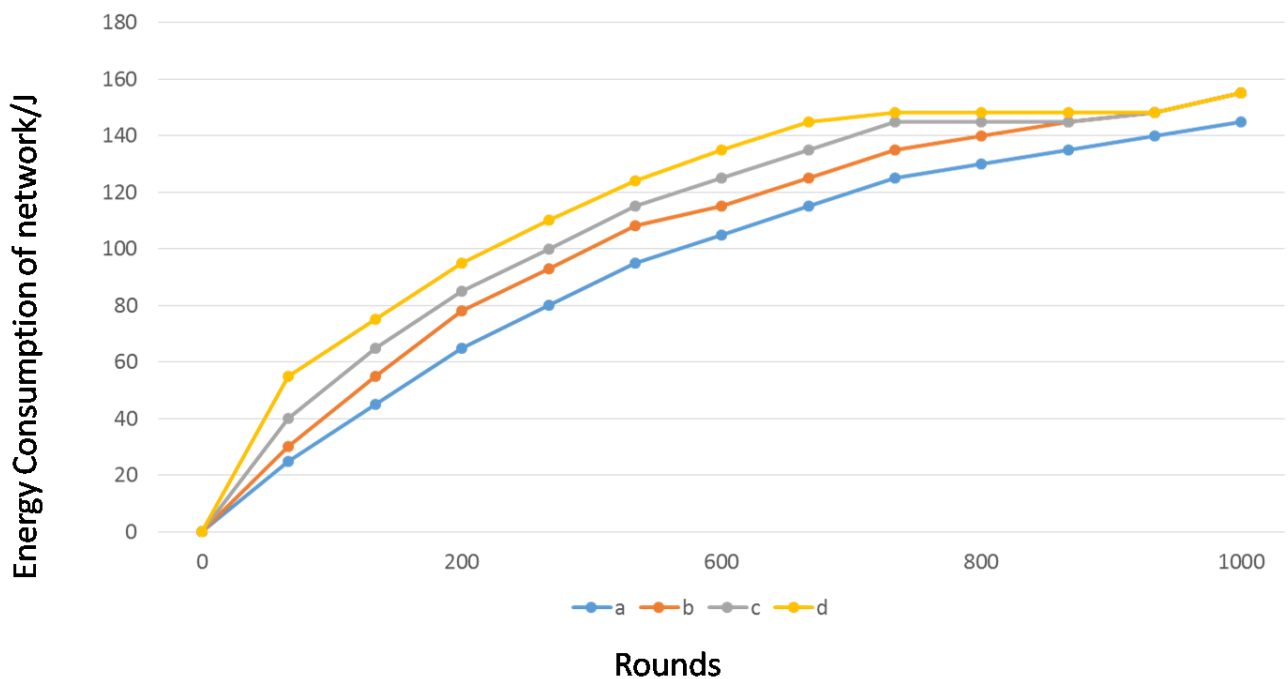

Fig.5. Network Energy Consumption

\section{Conclusion}

In this proposed work, a novel methodology to transmit data for marginal WSN with multipath reliable system. With completion of hierarchical clustering, it can be developed with network topology. Based on the routing information, first the original data is sub-divided into many packets which are transmitted via multi-paths in a simultaneous manner. When compared with other prevalent algorithms such as FRTSMC, DE-MRT and CBRACO, this protocol proves to be more reliable, has reduced data loss and has also improved the life cycle of the WSN.

\section{References}

[1] Mohanty, P., \& Kabat, M. R. (2016). Energy efficient reliable multi-path data transmission in WSN for healthcare application. International journal of wireless Information Networks, 23(2), 162-172.

[2] Zhang, J., Hu, P., Xie, F., Long, J., \& He, A. (2018). An energy efficient and reliable in-network data aggregation scheme for WSN. IEEE Access, 6, 71857-71870.

[3] Saeed, H., Ali, S., Rashid, S., Qaisar, S., \& Felemban, E. (2014, June). Reliable monitoring of oil and gas pipelines using wireless sensor network (WSN) - REMONG. In 2014 9th International Conference on System of Systems Engineering (SOSE) (pp. 230-235). IEEE.

[4] Zabin, F., Misra, S., Woungang, I., Rashvand, H. F., Ma, N. W., \& Ali, M. A. (2008). REEP: datacentric, energy-efficient and reliable routing protocol for wireless sensor networks. IET communications, 2(8), 995-1008.

[5] Di Marco, P., Park, P., Fischione, C., \& Johansson, K. H. (2010, May). TREnD: a timely, reliable, energy-efficient and dynamic wsn protocol for control applications. In 2010 IEEE International Conference on Communications (pp. 1-6). IEEE.

[6] Khalaf, O. I., \& Abdulsahib, G. M. (2020). Energy Efficient Routing and Reliable Data Transmission Protocol in WSN. Int. J. Advance Soft Compu. Appl, 12(3).

[7] Kamble, S., \& Dhope, T. (2016, May). Reliable routing data aggregation using efficient clustering in WSN. In 2016 International Conference on Advanced Communication Control and Computing Technologies (ICACCCT) (pp. 246-250). IEEE.

[8] Luo, J., Zhang, Z., Liu, C., \& Luo, H. (2018). Reliable and cooperative target tracking based on WSN and WiFi in indoor wireless networks. IEEE Access, 6, 24846-24855.

[9] Uthayakumar, J., Elhoseny, M., \& Shankar, K. (2020). Highly Reliable and Low-Complexity Image Compression Scheme Using Neighborhood Correlation Sequence Algorithm in WSN. IEEE Transactions on Reliability.

[10]Raj, J. S. (2019). ENERGY EFFICIENT SENSED DATA CONVEYANCE FOR SENSOR NETWORK UTILIZING HYBRID ALGORITHMS. Journal: IRO Journal on Sustainable Wireless Systems December, 2012(04), 235-246.

[11]Raj, J. S. (2019). ENERGY EFFICIENT SENSED DATA CONVEYANCE FOR SENSOR NETWORK UTILIZING HYBRID ALGORITHMS. Journal: IRO Journal on Sustainable Wireless Systems December, 2012(04), 235-246.

[12] Carvalho, C., Gomes, D. G., Agoulmine, N., \& De Souza, J. N. (2011). Improving prediction accuracy for WSN data reduction by applying multivariate spatio-temporal correlation. Sensors, 11(11), 1001010037. 